\title{
Acute phase proteins as diagnostic markers in horses with colic
}

Pihl, Tina; Scheepers, Elrien; Sanz, Macarena; Goddard, Amelia; Page, Patrick; Toft, Nils; Kjelgaard-Hansen, Mads; Andersen, Pia Haubro; Jacobsen, Stine

Published in:

Journal of Veterinary Emergency and Critical Care

DOI:

10.1111/vec. 12504

Publication date:

2016

Document version

Peer reviewed version

Citation for published version (APA):

Pihl, T., Scheepers, E., Sanz, M., Goddard, A., Page, P., Toft, N., Kjelgaard-Hansen, M., Andersen, P. H., \& Jacobsen, S. (2016). Acute phase proteins as diagnostic markers in horses with colic. Journal of Veterinary Emergency and Critical Care, 26(5), 664-674. https://doi.org/10.1111/vec.12504 

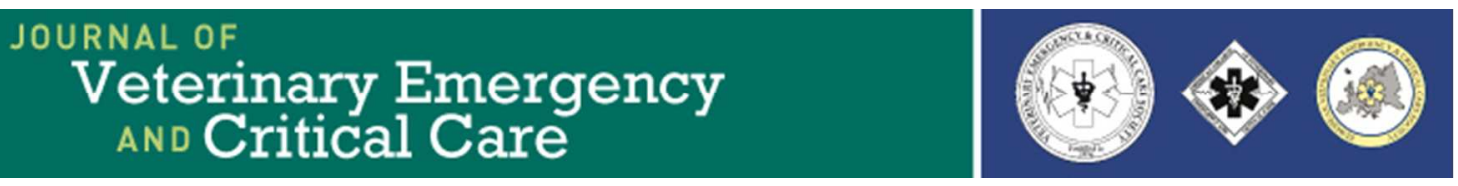

\section{Acute phase proteins as diagnostic markers in horses with colic}

\begin{tabular}{|r|l|}
\hline Journal: & Journal of Veterinary Emergency and Critical Care \\
\hline Manuscript ID: & JVECC-14-12-0001.R1 \\
\hline Wiley - Manuscript type: & Original Study \\
\hline Keyword: & $\begin{array}{l}\text { Serum Amyloid A, Haptoglobin, colic < Gastrointestinal < } \\
\text { Gastroenterology, Fibrinogen, Peritoneal fluid }\end{array}$ \\
\hline \multicolumn{2}{|l}{} \\
\hline
\end{tabular}

SCHOLARONE ${ }^{\text {tw }}$

Manuscripts 
1

2

3

4

\begin{abstract}
Objective - To investigate the diagnostic potential of acute phase proteins (serum amyloid A (SAA), haptoglobin and fibrinogen) measured in blood and peritoneal fluid for differentiating horses with inflammatory colic (entero-colitis and peritonitis) from those with surgical colic.

Design - Prospective observational multicenter study.

Setting - Two university referral hospitals.

Animals - Horses referred for severe acute abdominal pain to hospital $1(n=148)$ or hospital 2 $(n=78)$.
\end{abstract}

Intervention - Blood and peritoneal fluid samples collected at admission were used for acute phase protein measurement.

Measurements and Main Results - A multivariable logistic model including clinical parameters (lethargy, rectal temperature $>38^{\circ} \mathrm{C}$, normal rectal findings and gastric reflux of 5-10 L) recorded at admission was constructed from hospital 1 data. The ability of the model to correctly differentiate inflammatory from surgical colic was $86 \%$ determined as area under the receiver operating characteristic curve (AUC).

Adding blood parameters (white blood cell count (WBC), packed cell volume (PCV), total plasma protein, lactate, SAA, haptoglobin and fibrinogen) to the logistic model based on clinical parameters revealed that only SAA, WBC and fibrinogen improved the model. With SAA included in the model no additional blood parameters improved the model, and the final model had an AUC of $90 \%$. Addition of peritoneal fluid parameters (hemolysis, total protein, WBC, SAA, haptoglobin) did not improve the model significantly. When validated in hospital 2 data, the models had a satisfying integrity and diagnostic performance.

Conclusions - Evaluation of SAA in serum improved the ability to differentiate horses with acute inflammatory colic requiring medical treatment from horses with colic requiring surgical treatment, as it allowed an additional $4 \%$ of horses to be correctly classified into medical and surgical cases. Improved differentiation of these two groups of horses with colic may minimize the risk of unnecessary or delayed surgery. 
Words: 297 max 300

30

\section{Keywords:}

Acute phase proteins, serum amyloid A, haptoglobin, fibrinogen, peritoneal fluid, lactate, white blood cell count, colic, horse, diagnostic accuracy, surgery

32 


\section{Abbreviation list}

APP Acute phase proteins

AUC Area under the curve

DPJ Duodenitis-proximal jejunitis

Hp Haptoglobin

PF Peritoneal fluid

ROC Receiver operating characteristic

SAA Serum Amyloid A

\section{Introduction}

Horses with acute abdominal pain (colic) have traditionally been categorized into two major groups based on the required treatment namely horses where surgery is required and horses that can be treated medically ${ }^{1-6}$. This categorization is highly relevant because early surgical intervention optimizes the prognosis of the horse ${ }^{7}$. Horses in the acute stages of some inflammatory abdominal diseases such as duodenitis-proximal jejunitis (DPJ), acute colitis ${ }^{8,9}$ and peritonitis ${ }^{10}$, often present with the same severe signs of shock, pain, positive gastric reflux and/or changes in peritoneal fluid (PF) as horses with strangulations, displacements or severe impactions requiring surgical intervention. For horses with inflammatory colic, surgery will be an unnecessary burden and should be avoided ${ }^{11}$.

During inflammation, acute phase proteins (APPs) are released in circulation ${ }^{12}$. This is also the case in blood and PF of horses with colic, where higher concentrations of APPs are present in inflammatory diseases compared to simple obstructions or strangulations ${ }^{13-16}$. However, the diagnostic performance of APPs in differentiating inflammatory colic from colic that requires surgery has not been evaluated.

The objective of this study was to assess the value of APPs in the decision of medical versus surgical treatment in horses referred with severe acute abdominal pain to an equine hospital. A second objective was to evaluate the diagnostic value of adding PF evaluation to the information obtained from clinical examination and measurements of blood biomarkers. The study involved horses from two hospitals. The statistical models were developed based on data from one hospital and 
subsequently validated on data from the other hospital. This validation served to evaluate the robustness of the predictive models and to assess if they could be generalized to encompass hospitals with varying horse populations.

\section{Materials and Methods}

\section{Study design and population}

The study was a prospective observational multicenter study involving horses admitted with severe acute abdominal pain to XX (Hospital 1) from September 2008 to May 2011 and horses admitted to XX (Hospital 2) from August 2009 to December 2010. Horses were excluded if blood samples were not collected at admission, if the horse was less than 1 year old or pregnant with less than 1 month to term, or if a concurrent inflammatory disease unrelated to the abdomen was present (e.g. respiratory infections, hoof abscesses or wounds). Horses treated medically for simple obstructions, horses without a final diagnosis that responded to medical treatment, and horses with gastrointestinal ruptures were excluded from this study. Horses presenting with diarrhea or peritonitis without abdominal pain were also excluded from the study as the primary objective was to evaluate the diagnostic performance of the APPs in a population of horses that present a diagnostic challenge to the clinician.

The study was approved by the ethical boards of both hospitals. All data and samples were collected with the owner's permission as part of the routine diagnostic workup of the cases.

\section{Variables}

All horses underwent a full clinical examination immediately after admission, including rectal examination, nasogastric intubation, abdominocentesis, blood-gas analysis, fecal analysis for presence of sand, parasite eggs and larvae. Abdominal ultrasonography was performed in selected cases only. Clinical variables recorded were heart rate, respiratory rate, pain (none, mild, moderate, severe, lethargy), borborygmia (normal, decreased, ceased, increased), gastric reflux $(<5 \mathrm{~L}, 5-10 \mathrm{~L}$, $>10 \mathrm{~L}$ ), rectal findings (normal, impactions, dilatations, displacements), rectal temperature, capillary refill time (CRT), feces (normal, dry, soft, watery, none) and mucous membranes (pink, pale, congested, cyanotic). Blood was sampled for hematologic and serum biochemistry analyses. 
Variables measured in blood included serum amyloid A (SAA), haptoglobin (Hp), fibrinogen, iron, lactate, total white blood cell counts (WBC), total plasma protein (TPP) and packed cell volume (PCV). Variables measured in PF included SAA, Hp, total protein (TP), WBC and presence or absence of hemolysis. A final diagnosis was established based on all the information collected (excluding SAA, fibrinogen and $\mathrm{Hp}$ ) and, when available, surgical and post mortem findings. Demographic data, results of the clinical, hematologic, serum biochemical, PF and fecal analyses, pre-admission duration of colic $(0-12 \mathrm{~h}, 13-24 \mathrm{~h},>24 \mathrm{~h})$, required treatment (medical, surgical), and disease process (simple obstruction, strangulating obstruction, inflammatory, rupture, other and unknown) were recorded. Inflammatory diseases were defined as horses with DPJ, acute typhlo-colitis and acute peritonitis. Duodenitis-proximal jejunitis was diagnosed as horses with excessive gastric reflux $(>20 \mathrm{~L})$ over $>24 \mathrm{~h}$ that responded to medical treatment or where no concurrent mechanical obstruction was identified at surgery or necropsy ${ }^{17,18}$. Acute typhlo-colitis was diagnosed at necropsy and in horses that had severely compromised peripheral perfusion and developed diarrhea. Peritonitis was diagnosed as the primary disease in horses with PF WBC $>10 \times 10^{9} \mathrm{cells} / \mathrm{L}$ that responded to medical treatment, or had no apparent cause identified at surgery or post-mortem examination. Horses were assigned to either the medical or surgical group retrospectively by the principle investigator (THP) based on the final diagnosis.

\section{Collection and management of samples}

Blood and PF samples were collected and stored as described by Pihl et al. ${ }^{16}$. Briefly, unstabilized and citrate-stabilized blood samples and EDTA-stabilized PF samples were centrifuged at $2000 \mathrm{~g}$ for 10 minutes and supernatants stored at $-80^{\circ} \mathrm{C}$ until analysis for SAA, Hp and fibrinogen. Samples were shipped from hospital 2 to hospital 1 on dry ice with a professional cold chain operator ensuring constant temperature below $-80^{\circ} \mathrm{C}$.

\section{Laboratory analyses}

Packed cell volume and TPP were assessed by means of a Hawkley's microhaematocrit reader ${ }^{\mathrm{a}}$ and refractometry ${ }^{\mathrm{b}}$ respectively. Blood WBC were performed on two highly correlated automated instruments (ADVIA $120^{\mathrm{c}}$ in hospital 1 and ADVIA $2120^{\mathrm{d}}$ in hospital 2$)^{19}$. Plasma lactate was assessed 
within 10 minutes of collection by a spectrophotometric blood-gas analyzer ${ }^{\mathrm{e}}$ in hospital 1 and by a handheld lactate analyzer ${ }^{f}$ in hospital 2 . The handheld lactate analyzer had an acceptable correlation $(r=0.75)$ with a spectrophotometrically enzymatic kit at lactate concentrations $<10 \mathrm{mmol} / \mathrm{L}^{20}$. Serum amyloid $\mathrm{A}$ and $\mathrm{Hp}$ analyses of samples from both hospitals were performed in one batch at the laboratory of hospital 1 . Serum amyloid A was measured with the LZ SAA immunoturbidometric assay $^{\mathrm{g}}$ in an ADVIA $1800^{\mathrm{h}}$ as described by Jacobsen et al. $(2006)^{21}$. Haptoglobin was measured with the Phase Range Haptoglobin assay in an ADVIA $1800^{\mathrm{h}}$ as described by Pihl et al. $(2013)^{22}$. Fibrinogen was measured by the Clauss method in an automated coagulometric analyzer ACL $9000^{\mathrm{j}}$ as described by Andersen et al. (2012). Serum iron was measured by colorimetric spectrophotometry on an ADVIA $1650^{\mathrm{a}}$ as described by Andersen et al. $(2012)^{23}$.

\section{Statistical analyses}

The statistical outcome variable was presence or absence of inflammatory colic. Univariable logistic regression analysis of all registered variables from clinical findings, blood and PF analyses was used to identify variables eligible for inclusion in the multivariable models. For each variable the effect of pre-admission duration of colic was tested as an interaction term in both the univariable and multivariable analyses. Variables with $p<0.20$ in the univariable analyses were included in the multivariable models ${ }^{24}$. Manual backward elimination, followed by forward selection was used to construct the multivariable models. The criterion for retaining a variable in the final models was $p<$ $0.15^{25}$. Dummy variables for each category were constructed for the variables with multiple levels. Rectal temperature and CRT were changed to dichotomous variables (temperature deviation from $38^{\circ} \mathrm{C}$ and $\mathrm{CRT}$ to $\leq 2 \mathrm{~s}$ or $>2 \mathrm{~s}$ ) before entering the statistical models based on the biological definitions of normal values. A 'clinical model' was constructed by including only clinical variables. Variables measured in blood samples were then added to the clinical model; to construct a 'clinical + blood model'. Finally variables measured in PF were added to construct a 'clinical + blood + PF model'. This statistical approach was chosen to reflect the need of increasingly invasive methods to obtain samples (thus a clinically practical approach rather than a pure mathematical approach which would have identified the best markers overall). Data fit was evaluated by max rescaled $\mathrm{R}^{2}$ and Hosmer and Lemeshow goodness of fit. The influence of single observations on the models was tested by the 
residuals and covariate patterns of the regression diagnostics. The diagnostic performance of the resultant models was assessed by area under the receiver operating characteristics (ROC) curve (AUC) and by sensitivity and specificity at an optimal diagnostic cut-off point selected from the ROCcurve. A high specificity was prioritized in order to ensure that the horses identified as having inflammatory colic truly belonged to this group and did not require surgery, as misclassifying a horse with a surgical condition would potentially have more grave implications than vice versa.

In order to assess the robustness, each model was validated on data from hospital 2 in three steps, as described by Wiinberg et al. ${ }^{26}: 1$ ) Variables included in the model constructed on data from hospital 1 were tested for significant contribution to the model when applied to data from hospital $2 ; 2$ ) Variables excluded in the model were re-entered into the model by forward selection and their potential significant contribution to the model was evaluated; 3) Diagnostic performance of the model was assessed by applying the model and the defined cut-off value to data from hospital 2 . Demographic data was compared between the two disease groups overall and within each hospital with student's T-test for continuous variables (age and weight) and Chi-square-test for categorical variables (gender, breed) before logistic regression analyses. Confounding in the models was also tested by adding the demographic variables to the final models. Statistical analyses were done with SAS $9.2^{k}$.

\section{Results}

\section{Study population}

In total, 226 horses with acute severe colic were included in this study. The distribution of horses with inflammatory colic and surgical colic was similar in the two hospitals $(p=0.3)$, even though the distribution of specific diagnoses within each disease group varied (Table 1). There was no significant difference between age and gender in the two disease groups overall or within each hospital (Table 2). Horses with inflammatory colic weighed less than horses with surgical colic in hospital 1 and there was significantly more coldblooded horses in the inflammatory group than in the surgical group in hospital 2 (Table 2). 
Clinical model

Clinical variables identified by univariate analyses and included into the 'clinical model' were: pain,

Formatted: Normal, Line spacing: Double gastric reflux, rectal findings, feces, temperature above $38.0^{\circ} \mathrm{C}$, borborygmia and CRT (Table 3). Preadmission duration of colic did not influence any of the clinical variables evaluated. The final 'Clinical model' for prediction of inflammatory colic based on data from hospital 1 included lethargy, temperature increase from $38{ }^{\circ} \mathrm{C}$, gastric reflux 5-10 $\mathrm{L}$ and normal rectal findings (Table $\left.\underline{5} 4\right)$. All variables were positive predictors of inflammatory colic, except gastric reflux 5-10 L which was a negative predictor. The model was not confounded by demographical data (age, gender or breed) nor significantly influenced by single observations. The AUC was 0.86 (95 \% Cl: 0.79-0.93) (Figure 1). With a selected diagnostic cut-off at $p=0.576$ the diagnostic specificity was $98 \%$ and the sensitivity was $57 \%$. This means that $2 \%$ of horses requiring surgery were incorrectly classified as inflammatory (the false positive rate), while $43 \%$ of horses with inflammatory colic were incorrectly classified as requiring surgery (the false negative rate). Given the prevalence of inflammatory colic of $28 \%$ in Hospital 1, the positive predictive value of the 'Clinical model' was $93 \%$ and the negative predictive value $83 \%$ (Table $\underline{6} 5)$.

\section{Clinical model validation}

When the model developed in hospital 1 was evaluated using the population from hospital 2 all variables except gastric reflux 5-10 L were valid predictors. The model had an AUC of $0.75(95 \% \mathrm{Cl}$ : $0.61-0.89$ ) and at the selected cut-off at 0.576 the specificity was $96 \%$ and sensitivity $31 \%$ (Table 65). Gastric reflux $>10 \mathrm{~L}$ contributed significantly as a positive predictive variable with OR $=8.0$ (95\% $\mathrm{Cl}$ : 1.5-43.6) in hospital 2. The 'Clinical model' including gastric reflux $>10 \mathrm{~L}$ was therefore chosen as the best clinical model for prediction of inflammatory colic (Table 4).

\section{Effect of adding blood variables to the clinical model}

Blood variables added to the clinical model were PCV, SAA, fibrinogen, iron and WBC (Table 3). Serum amyloid A, fibrinogen, and WBC (combined with the duration of colic (D), WBC*D) all improved the clinical model with AUCs of the new models of $0.90,0.87$ and 0.86 respectively. However, serum 
SAA was associated with the greatest improvement in improved the clinical model-most and moreover, with SAA in the model (Figure 2). Nnone of the other measured biomarkers-measured in blood-significantly improved the model_(Figure 2). This model was not confounded by demographical data (age, gender or breed) nor significantly influenced by single observations.

This model had an AUC of 0.90 (95\% Cl: 0.84-0.96) (Figure 2). With a selected diagnostic cut-off at $p=0.5076$ the diagnostic specificity of the model was $98 \%$ and the sensitivity was $64 \%$ (Table $\underline{6} 5$ ). This means that in comparison to the clinical model the same number of horses $(2 \%)$ was falsely classified as inflammatory, whereas $35 \%$ horses instead of $43 \%$ were falsely classified as surgical. The positive predictive value of the 'Clinical+blood model' was $93 \%$ and the negative predictive value $\underline{87 \%}$.

When the model was validated on data from hospital 2, serum SAA was also found to significantly improve the clinical model in hospital 2, and no further variables had significant influence on predicting the probability of inflammatory colic. This model had an AUC of 0.84 (95 \% Cl: 0.72-0.95) (Figure 2), a specificity of $85 \%$ and a sensitivity of $63 \%$ (Table $\underline{5} 4$ and $\underline{6} 5$ ) in hospital 2 .

Figure 3 shows the actual concentrations of SAA in serum in the horses with inflammatory and surgical colic of both hospitals.

\section{Effect of further adding peritoneal fluid variables to the model}

Serum amyloid A was the only PF variable that was useful in differentiating inflammatory colic from surgical colic (Table 3). The duration of colic did not influence SAA in PF. The model was not improved by including SAA in PF. In hospital 2, similar results were obtained; therefore no model including PF variables was constructed.

\section{Discussion}

The overall aim of this study was to improve the differentiation between inflammatory and surgical colic, in order to select the optimal treatment regimen as early as possible. Assessment of serum SAA
Formatted: English (U.K.)

Formatted: Tab stops: $1.87 "$, Left 
added diagnostic information, and including this marker in the predictive models allowed $4 \%$ more horses to be correctly classified as inflammatory or surgical cases.

The approach in this study differed from previous diagnostic studies on prediction of need for surgery in two respects. Firstly, to reflect the importance of implications on possible falsely categorized horses, the model in this study aims at detecting horses NOT requiring surgery, as this would be valuable information to the clinician. When establishing and evaluating new diagnostic tools with highimpact outcome in a critical situation, a series of important biases are to be avoided if the results are to be useful ${ }^{27}$. Secondly, to avoid spectrum and limited-challenge biases only horses with severe colic, which was not easily assigned to either medical or surgical treatment based on the clinical examination alone, were included. This was done in order to evaluate the possible benefit of serum and PF APPs in the clinical setting, where it will be applied, as the trained clinician will most likely only revert to clinicopathological support in the acute decision-making, when the clinical examination fails to give a clear picture. Including horses where assignment to the groups in question was clear on clinical examination could have introduced increased risk of these biases.

Validating a model in another dataset is strongly recommended ${ }^{28,29}$ in order to test the integrity and performance of the model in another setting than the one in which it was constructed. The prevalence of inflammatory colic was similar between the two hospitals (28\% for hospital 1 and $22 \%$ for hospital 2). Even though the distribution of the specific diagnoses within the two groups of colic varied between hospitals, the integrity of the models was high, demonstrated by the fact that the same variables had significant diagnostic value in both hospitals. Interestingly, the variable "large volume reflux (> $10 \mathrm{~L})$ " was a better predictor in hospital 2 than low volume $(5-10 \mathrm{~L})$ reflux. A possible explanation for this is that hospital 2 had more horses with DPJ, a condition characterized by large volume gastric reflux.

The clinical variables included in the model are in agreement with earlier studies on factors predicting the need for surgery ${ }^{1,2,11}$. However, in contrast to other studies that have shown that assessment of PF variables such as total protein and hemolysis may provide useful diagnostic information $^{2,4,30}$, the present study did not detect any further advantages of adding PF variables to the clinical and blood variables. The reason for this finding probably relates to our inclusion criteria, where only severe colic cases were included. Previous studies included less severe abdominal disease, such as simple large intestinal obstructions, when comparing medical and surgical abdominal conditions. 
Obstructions and other mild medical abdominal disease often cause little changes to PF, and studies with these cases included often revealed great differences in composition of PF between horses with medical and surgical colic. The advantage of the design of the present study was that it took into account the clinical situation where abdominocentesis is reserved for cases where no other decision making tools are available.

One limitation to this study was the lack of PF evaluation in all horses and the lack of WBC counts in all PF samples. In addition, only data collected at admission was evaluated, since only a few horses requiring surgery had a second PF sample collected. Changes in PF lactate have recently been reported to be valuable in identifying horses requiring surgery when serial measures were performed $^{31}$. Future studies should therefore include serial measurements of blood and PF biomarkers in order to improve the diagnostic and prognostic performance of the markers.

Other studies evaluating models predicting the need for surgery have generally overestimated the need for surgery ${ }^{1,2,30}$. The diseases most often misclassified as surgical are those of inflammatory origin. With an AUC of 0.9 in hospital 1 and 0.84 in hospital 2, the number of horses misclassified with the model that included serum SAA was lower than with previous models ${ }^{1,2,30}$.

Serum Amyloid A has been measured in several studies investigating various inflammatory diseases in horses ${ }^{12}$. In this study, serum SAA was found to have significant diagnostic capacity in differentiating inflammatory from surgical colic. Including assessment of serum SAA allowed an additional $4 \%$ of horses to be classified correctly as needing either medical or surgical treatment.

Duration of disease has been suggested to be an important factor when evaluating APPs and lactate concentrations in horses with colic $^{16}$. Adding pre-admission duration of colic to the model did not change the diagnostic performance of SAA or the model significantly. This is likely the result of SAA being a fast reacting biomarker that is significantly increased in horses with colic already at durations of 5-12 $\mathrm{h}^{16}$. Fibrinogen, $\mathrm{Hp}$ and WBC increase later and thus have a stronger dependency on duration ${ }^{16}$. When evaluated as single variables, fibrinogen and WBC had significant diagnostic capacity, but when added to the clinical model they did not perform as well as SAA, despite taking duration of colic into consideration. This might be because of the large span of the duration intervals used in this study or because of the often inaccurate estimation of colic duration given by the owner. 

will only be useful as a diagnostic marker if the analysis can be performed on a single sample basis, within a short time and at a reasonable price ${ }^{32}$. Such point of care tests are currently commercially available ${ }^{33}$.

In conclusion, adding measurement of serum SAA to the clinical assessment of horses with severe colic improved the identification of horses with acute inflammatory colic that did not require surgery. Including SAA in the evaluation of horses with abdominal disease can potentially minimize the risk of unnecessary or delayed surgery, and a $4 \%$ improvement in diagnosis is relevant in a specialized referral hospital. Validation of the model on an entirely different colic population showed that the model was valid in different hospital settings. 
290

\section{Footnotes}

${ }^{\mathrm{a}}$ Hawksley Medical and Laboratory Equipment, Lancing, UK

${ }^{\mathrm{b}}$ Atago Sur-Ne Clinical Refractometer, ATAGO CO., LTD, Tokyo, Japan

${ }^{\mathrm{C} A D V I A} 120$, Bayer A/S ,Lyngby, Denmark

${ }^{\text {d} A D V I A ~ 2120, ~ S i e m e n s ~ H e a l t h ~ C a r e ~ D i a g n o s t i c s ~ I n c . ~, ~ N Y, ~ U S A ~}$

${ }^{\mathrm{e}}$ Radiometer ABL725, Radiometer Medical ApS, Brønshøj, Denmark

${ }^{f}$ Accusport Blood Lactate Analyzer, Roche Diagnostics, Basel, Switzerland

${ }^{9}$ EIKEN Chemical Co. Ltd., Tokyo, Japan

${ }^{\text {h} A D V I A ~} 1800$ Chemistry System, Siemens Health Care Diagnostics Inc., IL, USA

'Tridelta Development Ltd., Ireland

${ }^{\mathrm{j} A C L} 9000$, Instrumentation Laboratory

${ }^{\mathrm{k}}$ SAS Institute SAS Inc., NC, USA

\section{References}

1. Parry BW, Gay CC and Anderson GA. Assessment of the necessity for surgical intervention in cases of equine colic: a retrospective study. Equine Vet. J. 1983;15(3):216-221.

2. Thoefner MB, Ersboll BK, Jansson $\mathrm{N}$ and Hesselholt M. Diagnostic decision rule for support in clinical assessment of the need for surgical intervention in horses with acute abdominal pain. Can J Vet Res 2003;67(1):20-29. 
3. Reeves MJ, Curtis CR, Salman MD et al. Multivariable Prediction Model for the Need for Surgery in Horses with Colic. Am J Vet Res 1991;52(11):1903-1907.

4. Freden GO, Provost PJ and Rand WM. Reliability of using results of abdominal fluid analysis to determine treatment and predict lesion type and outcome for horses with colic: 218 cases (19911994). J Am Vet Med Assoc 1998;213(7):1012-1015

5. Matthews S, Dart AJ, Reid SWJ et al. Predictive values, sensitivity and specificity of abdominal fluid variables in determining the need for surgery in horses with an acute abdominal crisis. Aus Vet J 2002;80(3):132-136.

6. White NA, Elward A, Moga KS et al. Use of web-based data collection to evaluate analgesic administration and the decision for surgery in horses with colic. Equine Vet J 2005;37(4):347-350.

7. Mair TS, Smith LJ and Sherlock CE. Evidence-based gastrointestinal surgery in horses. Veterinary Vet Clin N Am - Equine 2007;23(2):267-292.

8. Feary DJ and Hassel DM. Enteritis and Colitis in Horses. Vet Clin N Am - Equine 2006;22(2):437479.

9. Southwood LL. Acute Abdomen. Clin Tech Equine P 2006;5(2):112-126.

10. Parry BW. Use of clinical pathology in evaluation of horses with colic. Vet Clin N Am - Equine $1987 ; 3(3): 529-542$.

11. Johnston JK and Morris DD. Comparison of Duodenitis Proximal Jejunitis and Small IntestinalObstruction in Horses - 68 Cases (1977-1985). J Am Vet Med Assoc 1987;191(7):849-854. 
12. Jacobsen $\mathrm{S}$ and Andersen $\mathrm{PH}$. The acute phase protein serum amyloid $\mathrm{A}(\mathrm{SAA})$ as a marker of inflammation in horses. Equine Vet Educ 2007;19(1):38-46.

13. Vandenplas ML, Moore JN, Barton $\mathrm{MH}$ et al. Concentrations of serum amyloid $\mathrm{A}$ and lipopolysaccharide-binding protein in horses with colic. Am J Vet Res 2005;66(9):1509-1516.

14. Forbes G, Sorich E, Nath LC et al. Activin A as a novel biomarker of equine inflammatory abdominal disease: preliminary findings. Vet J 2011;190(2):154-156.

15. Prasse KW, Topper MJ, Moore JN and Welles EG. Analysis of hemostasis in horses with colic. J Am Vet Med Assoc 1993;203(5):685-693.

16. Pihl TH, Scheepers E, Goddard A et al. Influence of disease process and duration on acute phase proteins in serum and peritoneal fluid of horses with acute abdominal pain. $\mathrm{J}$ Vet Intern Med 201며;(In press) doi 10.1111/jvim.12542

17. Underwood C, Southwood LL, McKeown KP and Knight D. Complications and survival associated with surgical compared with medical management of horses with duodenitis-proximal jejunitis. Equine Vet J 2008;40(4):373-378.

18. Cohen ND, Toby E, Roussel AJ et al. Are feeding practices associated with duodenitis-proximal jejunitis? Equine Vet J 2006;38(6):526-531.

19. Harris N, Jou JM, Devoto G et al. Performance evaluation of the ADVIA 2120 hematology analyzer: an international multicenter clinical trial. Lab Hematol. 2005;11(1):62-70. 
20. Schulman ML, Nurton JP and Guthrie AJ. Use of the Accusport semi-automated analyser to determine blood lactate as an aid in the clinical assessment of horses with colic. J S Afr Vet Assoc 2001;72(1):12-17.

21. Jacobsen S, Kjelgaard-Hansen M, Petersen HH and Jensen AL. Evaluation of a commercially available human serum amyloid A (SAA) turbidometric immunoassay for determination of equine SAA concentrations. Vet J 2006;172(2):315-319.

22. Pihl TH, Andersen $\mathrm{PH}$, Kjelgaard-Hansen $\mathrm{M}$ et al. Serum amyloid $\mathrm{A}$ and haptoglobin concentrations in serum and peritoneal fluid of healthy horses and horses with acute abdominal pain. Vet Clin Pathol 2013;42(2):177-183.

23. Andersen SA, Petersen $\mathrm{HH}$, Ersbøll AK et al. Vaccination elicits a prominent acute phase response in horses. The Vet J 2012;191(2):199-202.

24. Altman, D. G. (1991) Relation between several variables. In: Practical statistics for medical research, 1th edn., Chapman \& Hall, Boca Raton, FL. pp. 325-364.

25. Hosmer DW, Hosmer T, Le CS and Lemeshow S. A comparison of goodness-of-fit tests for the logistic regression model. Stat Med 1997;16(9):965-980.

26. Wiinberg B, Jensen AL, Johansson PI et al. Development of a model based scoring system for diagnosis of canine disseminated intravascular coagulation with independent assessment of sensitivity and specificity. Vet J 2010;185(3):292-298.

27. Bossuyt PM, Reitsma JB, Bruns DE et al. The STARD statement for reporting studies of diagnostic accuracy: explanation and elaboration. Clin Chem 2003;49(1):7-18. 
28. Lemeshow S, Teres D, Pastides $\mathrm{H}$ et al. A method for predicting survival and mortality of ICU patients using objectively derived weights. Crit Care Med 1985;13(7):519-525.

29. Reeves MJ, Curtis CR, Salman MD et al. Validation of Logistic-Regression Models Used in the Assessment of Prognosis and the Need for Surgery in Equine Colic Patients. Prev Vet Med 1992;13(3):155-172.

30. Ducharme NG, Pascoe PJ, Lumsden JH and Ducharme GR. A computer-derived protocol to aid in selecting medical versus surgical treatment of horses with abdominal pain. Equine Vet $\mathrm{J}$ 1989;21(6):447-450.

31. Peloso JG and Cohen ND. Use of serial measurements of peritoneal fluid lactate concentration to identify strangulating intestinal lesions in referred horses with signs of colic. J Am Vet Med Assoc 2012;240(10):1208-1217.

32. Kjelgaard-Hansen M and Jacobsen S. Assay validation and diagnostic applications of major acute-phase protein testing in companion animals. Clin Lab Med 2011;31(1):51-70.

33. Jacobsen $S$ and Kjelgaard-Hansen M. Evaluation of a commercially available apparatus for measuring the acute phase protein serum amyloid A in horses. Vet Rec 2008;163(11):327-330. 
Figure legends

Figure 1.

A) The best model with clinical parameters predicting the probability of inflammatory colic in horses referred to a referral clinic (hospital 1 ) with acute, severe abdominal disease. The model included the

clinical variables lethargy, rectal temperature above $38.0{ }^{\circ} \mathrm{C}$, gastric reflux $5-10 \mathrm{~L}$, and normal rectal findings. With an area under the receiver operating characteristic (ROC) curve (AUC) of 0.86 the model correctly diagnosed inflammatory colic and surgical colic in $86 \%$ of the horses evaluated. B) Validation of the Clinical model in another referral clinic (hospital 2) identified the same variables as significant predictors (except gastric reflux, where large volume reflux ( $>10 \mathrm{~L}$ ) was superior to low volume reflux (5-10 L)). The AUC is 0.82 in hospital 2.

Figure 2.

A) The best model with clinical and blood parameters constructed in hospital 1 included only SAA in serum in addition to the clinical variables. SAA in serum improved the AUC to 0.90 from 0.86 . B) SAA in serum likewise improved the clinical model in hospital 2 increasing the AUC to 0.84 from 0.82 . With SAA in serum in the model no further blood or PF variables significantly improved the model. 
19

407

408
Tables

Table 1. Diagnoses of horses included in the study from hospital 1 and 2.

\begin{tabular}{|c|c|c|}
\hline & Hospital 1 & Hospital 2 \\
\hline Inflammatory (\%) & $42(28 \%)$ & $17(22 \%)$ \\
\hline Duodenitis-proximal jejunitis & $8(19 \%)$ & $6(35.3 \%)$ \\
\hline Enterocolitis & $18(43 \%)$ & $6(35.3 \%)$ \\
\hline Peritonitis & $16(38 \%)$ & $5(29.4 \%)$ \\
\hline Surgical (\%) & $106(72 \%)$ & $61(78 \%)$ \\
\hline Strangulating obstructions & $64(59 \%)$ & $25(41 \%)$ \\
\hline Small intestinal strangulations & $32(50 \%)$ & $15(60 \%)$ \\
\hline Verminous thromboembolic infarct & $12(19 \%)$ & $0(0.0 \%)$ \\
\hline Large colon torsions or strangulations & $20(31 \%)$ & $10(40.0 \%)$ \\
\hline Simple obstructions & $40(38 \%)$ & $35(57 \%)$ \\
\hline Ascending colon impactions & $6(15 \%)$ & $5(14 \%)$ \\
\hline Descending colon impactions & $3(7.5 \%)$ & $2(6 \%)$ \\
\hline Caecum impactions & $2(5 \%)$ & $1(3 \%)$ \\
\hline Caecum tympani & 0 & $1(3 \%)$ \\
\hline Colon displacements without strangulation & $22(55 \%)$ & $18(51 \%)$ \\
\hline Small intestinal impactions & $7(17.5 \%)$ & $8(23 \%)$ \\
\hline Extra-enteral (testicular torsion) & 0 & $1(2 \%)$ \\
\hline Miscellaneous & $2(2 \%)$ & $1(2 \%)$ \\
\hline
\end{tabular}


Table 2. Demographic data of horses included in the study from hospital 1 and 2.

\begin{tabular}{|c|c|c|c|}
\hline & Inflammatory colic & Surgical colic & p-value \\
\hline Horses (n) & 59 & 167 & \\
\hline Hospital 1 & 42 & 106 & \\
\hline Hospital 2 & 17 & 61 & \\
\hline Age (years) & $10.3(8.7-11.8)$ & $9.4(8.6-10.2)$ & 0.3 \\
\hline Hospital 1 & $10.7(8.8-12.5)$ & $9.9(8.9-11.0)$ & 0.5 \\
\hline Hospital 2 & $9.3(6.4-12.2)$ & $8.4(7.4-9.5)$ & 0.6 \\
\hline Weight (kg) & $449(417-481)$ & $495(478-512)$ & 0.015 \\
\hline Hospital 1 & $445(406-485)$ & $502(477-528)$ & 0.02 \\
\hline Hospital 2 & $459(397-521)$ & $482(465-498)$ & 0.5 \\
\hline Gender (\%) & & & 0.8 \\
\hline Hospital 1 & & & 0.73 \\
\hline Mares & $20(48 \%)$ & $45(42 \%)$ & \\
\hline Stallions & $3(7 \%)$ & $6(6 \%)$ & \\
\hline Geldings & $19(45 \%)$ & $55(52 \%)$ & \\
\hline Hospital 2 & & & 0.65 \\
\hline Mares & $6(35 \%)$ & $28(46 \%)$ & \\
\hline Stallions & $3(18 \%)$ & $7(11 \%)$ & \\
\hline Geldings & $8(47 \%)$ & $26(43 \%)$ & \\
\hline Breeds (\%) & & & 0.006 \\
\hline Hospital 1 & & & 0.35 \\
\hline "Warm blooded"" & $22(52 \%)$ & $66(62 \%)$ & \\
\hline "Cold blooded"§ & $20(48 \%)$ & $40(38 \%)$ & \\
\hline Hospital 2 & & & 0.0014 \\
\hline "Warm blooded" & $10(59 \%)$ & $57(93 \%)$ & \\
\hline "Cold blooded"§ & $7(41 \%)$ & $4(7 \%)$ & \\
\hline
\end{tabular}


413 ":"Warm blooded" includes Warmbloods, Standardbreds, Thoroughbreds, Arabians and Western 414 breeds.

415 §. "Cold blooded" includes Icelandic horses, ponies and draught horses. 
Table 3. The predicitive value of clinical variables for inflammatory colic

\begin{tabular}{|c|c|c|c|c|c|}
\hline & $\mathbf{n}$ & AUC & $\begin{array}{c}\text { Crude OR } \\
\text { infectioninfla } \\
\text { mmation }\end{array}$ & $95 \% \mathrm{CI}$ OR & p-value \\
\hline \multicolumn{6}{|c|}{ Clinical categorical variables } \\
\hline Duration & 135 & 0.57 & & & 0.36 \\
\hline$>24$ & 43 & & 1.86 & $0.80-4.34$ & \\
\hline $13-24$ & 30 & & 1.34 & $0.51-3-55$ & \\
\hline $0-12 \mathrm{~h}$ & 62 & & Reference & - & \\
\hline Pain & 143 & 0.71 & & & 0.15 \\
\hline Lethargy & 25 & & 2.4 & $0.8-7.0$ & 0.9 \\
\hline Severe & 23 & & $<0.001$ & $<0.001->999$ & 0.9 \\
\hline Moderate & 17 & & 0.3 & $0.06-1.5$ & 1.0 \\
\hline Mild & 46 & & 1.1 & $0.4-2.8$ & 0.9 \\
\hline No & 32 & & Reference & & \\
\hline Borborygmia & 145 & 0.58 & & & 0.18 \\
\hline Increased & 6 & & 3.0 & $0.26-35.3$ & 0.1 \\
\hline Ceased & 52 & & 0.61 & $0.92-4.01$ & 0.3 \\
\hline Decreased & 82 & & 0.45 & $0.07-2.91$ & 0.05 \\
\hline Normal & 5 & & Reference & - & \\
\hline Gastric reflux & 141 & 0.59 & & & 0.093 \\
\hline$>10 \mathrm{~L}$ & 17 & & 0.82 & $0.27-2.52$ & 0.3 \\
\hline $5-10 \mathrm{~L}$ & 23 & & 0.19 & $0.04-0.85$ & 0.05 \\
\hline$<5 \mathrm{~L}$ & 101 & & Reference & & \\
\hline Rectal findings & 139 & 0.78 & & & $<0.0001$ \\
\hline Displacements & 37 & & 0.03 & $0.007-0.12$ & 0.0027 \\
\hline Dilated intestines & 51 & & 0.05 & $0.015-0.17$ & 0.03 \\
\hline Obstipation & 25 & & 0.09 & $0.025-0.34$ & 0.7 \\
\hline
\end{tabular}




\begin{tabular}{|c|c|c|c|c|c|}
\hline Normal & 26 & & 1 & - & \\
\hline Feces & 135 & 0.58 & & & 0.13 \\
\hline Soft or watery & 16 & & 2.25 & $0.62-8.14$ & 0.08 \\
\hline Dry or no & 93 & & 0.74 & $0.28-1.93$ & 0.08 \\
\hline Normal & 26 & & Reference & - & \\
\hline Mucous membranes & 140 & 0.58 & & & 0.43 \\
\hline Cyanotic & 3 & & 0.79 & $0.15-4.18$ & 0.6 \\
\hline Red & 41 & & 1.78 & $0.78-4.07$ & 0.1 \\
\hline Pale & 22 & & 0.82 & $0.26-2.54$ & 0.6 \\
\hline Pink & 68 & & Reference & - & \\
\hline $\begin{array}{l}\text { Capillary Refill Time } \\
\text { (CRT) }\end{array}$ & 128 & 0.57 & & & 0.16 \\
\hline Prolonged (>2s.) & 68 & & 1.75 & $0.81-3.79$ & \\
\hline Normal ( $\leq 2 \mathrm{~s}$.) & 60 & & Reference & & \\
\hline \multicolumn{6}{|c|}{ Clinical continuous variables } \\
\hline Heart rate (beats/min) & 147 & 0.53 & 1.004 & $0.98-1.03$ & 0.71 \\
\hline Temperature $-38\left({ }^{\circ} \mathrm{C}\right)$ & 141 & 0.64 & 1.92 & $1.16-3.16$ & 0.01 \\
\hline
\end{tabular}

419 Explanatory note: An odds ratio $(O R)=1$ represents no association, $O R>1$ represents a predisposing association where as an $\mathrm{OR}<1$ represents a protective association. The OR describes the increased risk for an increase in one unit of the investigated variable. Area under the curve (AUC) describes the probability of correctly classifying an inflammatory case as such $(1.00=100 \%, 0.5=$ no better than chance). 
Table 4. The predicitive value of blood and peritoneal fluid variables for inflammatory colic

\begin{tabular}{|c|c|c|c|c|c|}
\hline & $\mathbf{n}$ & AUC & $\begin{array}{c}\text { Crude OR } \\
\text { infectioninfla } \\
\text { mmation }\end{array}$ & $95 \% \mathrm{CI}$ OR & p-value \\
\hline \multicolumn{6}{|l|}{ Blood variables } \\
\hline Packed cell volume (\%) & 147 & 0.61 & 1.04 & $1.00-1.08$ & 0.033 \\
\hline Plasma protein (g/L) & 146 & 0.53 & 0.99 & $0.96-1.02$ & 0.47 \\
\hline WBC $\left(10^{y}\right.$ cells $\left./ L\right)$ & 128 & 0.58 & 0.93 & $0.83-1.04$ & 0.18 \\
\hline Lactate $(\mathrm{mmol} / \mathrm{L})$ & 146 & 0.50 & 1.04 & $0.96-1.13$ & 0.33 \\
\hline Serum amyloid A (mg/L) & 148 & 0.69 & 1.001 & $1.00-1.001$ & 0.0005 \\
\hline Haptoglobin (mg/L) & 147 & 0.53 & 1.000 & $1.00-1.00$ & 0.36 \\
\hline Fibrinogen (g/L) & 124 & 0.61 & 1.30 & $1.04-1.64$ & 0.023 \\
\hline Iron $(\mu \mathrm{mol} / \mathrm{L})$ & 125 & 0.62 & 0.97 & $0.93-1.01$ & 0.09 \\
\hline \multicolumn{6}{|l|}{ Peritoneal fluid variables } \\
\hline Hemolysis & 135 & 0.53 & & & 0.47 \\
\hline Hemolysis & 39 & & 0.73 & $0.31-1.73$ & \\
\hline No hemolysis & 96 & & Reference & - & \\
\hline WBC $\left(10^{9}\right.$ cells $\left./ L\right)$ & 58 & 0.68 & 1.002 & $0.99-1.01$ & 0.60 \\
\hline Total protein_(g/L) & 140 & 0.52 & 1.002 & $0.98-1.026$ & 0.86 \\
\hline Serum amyloid $A$ (mg/L) & 134 & 0.62 & 1.001 & $1.00-1.003$ & 0.0087 \\
\hline Haptoglobin (mg/L) & 135 & 0.52 & 1.000 & $1.00-1.001$ & 0.51 \\
\hline \multicolumn{6}{|c|}{$\begin{array}{l}\text { Explanatory note: An odds ratio }(O R)=1 \text { represents no association, OR }>1 \text { represents a predisposing } \\
\text { association where as an } O R<1 \text { represents a protective association. The OR describes the increased } \\
\text { risk for an increase in one unit of the investigated variable. Area under the curve (AUC) describes the } \\
\text { probability of correctly classifying an inflammatory case as such }(1.00=100 \%, 0.5=\text { no better than }\end{array}$} \\
\hline
\end{tabular}


Table 54. Results of multivariable logistic regression analysis showing the association between inflammatory colic and variables included in the 'clinical model' and 'clinical+blood model' based on data from hospital 1 and validated in hospital 2.

\begin{tabular}{|c|c|c|c|c|c|}
\hline & $\mathbf{n}$ & AUC & $\begin{array}{l}\text { Adjusted } \\
\text { OR }\end{array}$ & $\begin{array}{l}95 \% \mathrm{Cl} \\
\text { Adj. OR }\end{array}$ & $p$-value \\
\hline Clinical model & 141 & 0.86 & & & \\
\hline Normal rectal findings & & & 38.5 & $7.4-199$ & \\
\hline Lethargy & & & 5.6 & $1.4-22.7$ & 0.02 \\
\hline Temperature $-38\left({ }^{\circ} \mathrm{C}\right)$ & & & 1.8 & $1.0-3.5$ & 0.06 \\
\hline Gastric reflux 5-10L & & & 0,06 & $0,005-0,7$ & 0,03 \\
\hline Gastric reflux $>10 \mathrm{~L}$ & & & 1.5 & $0.4-5.2$ & 0.6 \\
\hline Clinical + blood model & 141 & 0.90 & & & \\
\hline Normal rectal findings & & & 37.3 & $6.9-202$ & $<0.001$ \\
\hline Lethargy & & & 6.7 & $1.6-28.4$ & 0.01 \\
\hline Temperature-38 $\left({ }^{\circ} \mathrm{C}\right)$ & & & 1.7 & $0.9-3.3$ & 0.08 \\
\hline Gastric reflux 5-10L & & & 0.07 & $0.06-0.7$ & 0.03 \\
\hline Gastric reflux $>10 \mathrm{~L}$ & & & 1.9 & $0.5-7.3$ & 0.3 \\
\hline $\begin{array}{l}\text { Serum amyloid } A \text { in } \\
\text { serum }(100 \mathrm{mg} / \mathrm{L})\end{array}$ & & & 1.06 & $1.01-1.1$ & 0.01 \\
\hline
\end{tabular}

Explanatory note: Area under the ROC curve (AUC) describes the probability of correctly classifying an inflammatoryectious case as such $(1.00=100 \%, 0.5=$ no better than chance $)$. An odds ratio $(O R)=$ 1 represents no association, OR $>1$ represents a predisposing association where as an $\mathrm{OR}<1$ represents a protective association. For continuous variables the OR describes the increased risk for an increase in one unit of the investigated variable. For SAA the OR is given per $100 \mathrm{mg} / \mathrm{L}$ increase. 
Table 65 . Diagnostic performance of the $\mathbf{2}$ models developed in hospital 1 and validated in hospital 2.

\begin{tabular}{|c|c|c|c|c|c|c|c|c|}
\hline & AUC & $95 \% \mathrm{Cl}$ & Se (\%) & $\begin{array}{l}\text { Sp } \\
(\%)\end{array}$ & $\begin{array}{l}\text { FPR } \\
(\%)\end{array}$ & $\begin{array}{l}\text { PPV } \\
\text { (\%) }\end{array}$ & $\begin{array}{l}\text { NPV } \\
\text { (\%) }\end{array}$ & $\begin{array}{l}\text { LR+ } \\
\text { (\%) }\end{array}$ \\
\hline \multicolumn{9}{|c|}{ Clinical model $^{*}$} \\
\hline Hospital 1 & 0.86 & $0.79-0.93$ & 57 & 98 & 2 & 93 & 85 & 28.5 \\
\hline Hospital 2 & 0.82 & $0.71-0.93$ & 38 & 90 & 10 & 52 & 84 & 3,89 \\
\hline \multicolumn{9}{|c|}{ Clinical + Blood model ${ }^{\S}$} \\
\hline Hospital 1 & 0.90 & $0.84-0.96$ & 64 & 98 & 2 & 93 & 87 & 32 \\
\hline Hospital 2 & 0.84 & $0.72-0.95$ & 63 & 85 & 15 & 54 & 89 & 4.2 \\
\hline
\end{tabular}

441

Explanatory note::

${ }^{*}$ Clinical model' (Selected cut off $=0.5633$ for a positive test):

$Y=-0.094+0.86^{*}$ lethargy $+0.59^{*}$ (temperature-38.0 $)+\left(-1.38^{*}\right.$ gastric reflux $\left.5-10 \mathrm{~L}\right)+0.19^{*}$ gastric reflux $>10 \mathrm{~L}+1.82^{*} \mathrm{normal}$ rectal finding.

§ Clinical + blood model $($ Selected cut off $=0.5076$ for a positive test $)$ :

$\mathrm{Y}=-0.23+0.95^{*}$ lethargy $+0.56^{*}$ (temperature-38.0) $+\left(-1.34^{*}\right.$ gastric reflux $\left.5-10 \mathrm{~L}\right)+0.33^{*}$ gastric reflux $>10 \mathrm{~L}$ $+1.81 *$ Normal_rectal $+0.00061 *$ SAAserum.

$A \cup C=$ the percentages of horses correctly classified as diseased (inflammatory) or not (surgical) by the test.

$\mathrm{Se}=$ Sensitivity $=$ Detection rate $=$ the proportion of disease positives (inflammatory) correctly classified by the test

$\mathrm{Sp}=$ Specificity $=$ the proportion of disease negative (surgical colic) correctly classified by the test.

$\mathrm{FPR}=$ False positive rate $=(1-\mathrm{sp})=$ proportion of horses falsely classified as diseased (inflammatory) by the test.

PPV $=$ Positive predictive value $=$ the probability that a positive test is reflecting presence of disease (inflammatory).

NPV $=$ Negative predictive value $=$ the probability that a negative test is reflecting absence of the disease (surgical).

$\mathrm{LR}+=$ Likelihood ratio $=$ the likelihood ratio of a positive test results in an animal with disease (inflammatory) 


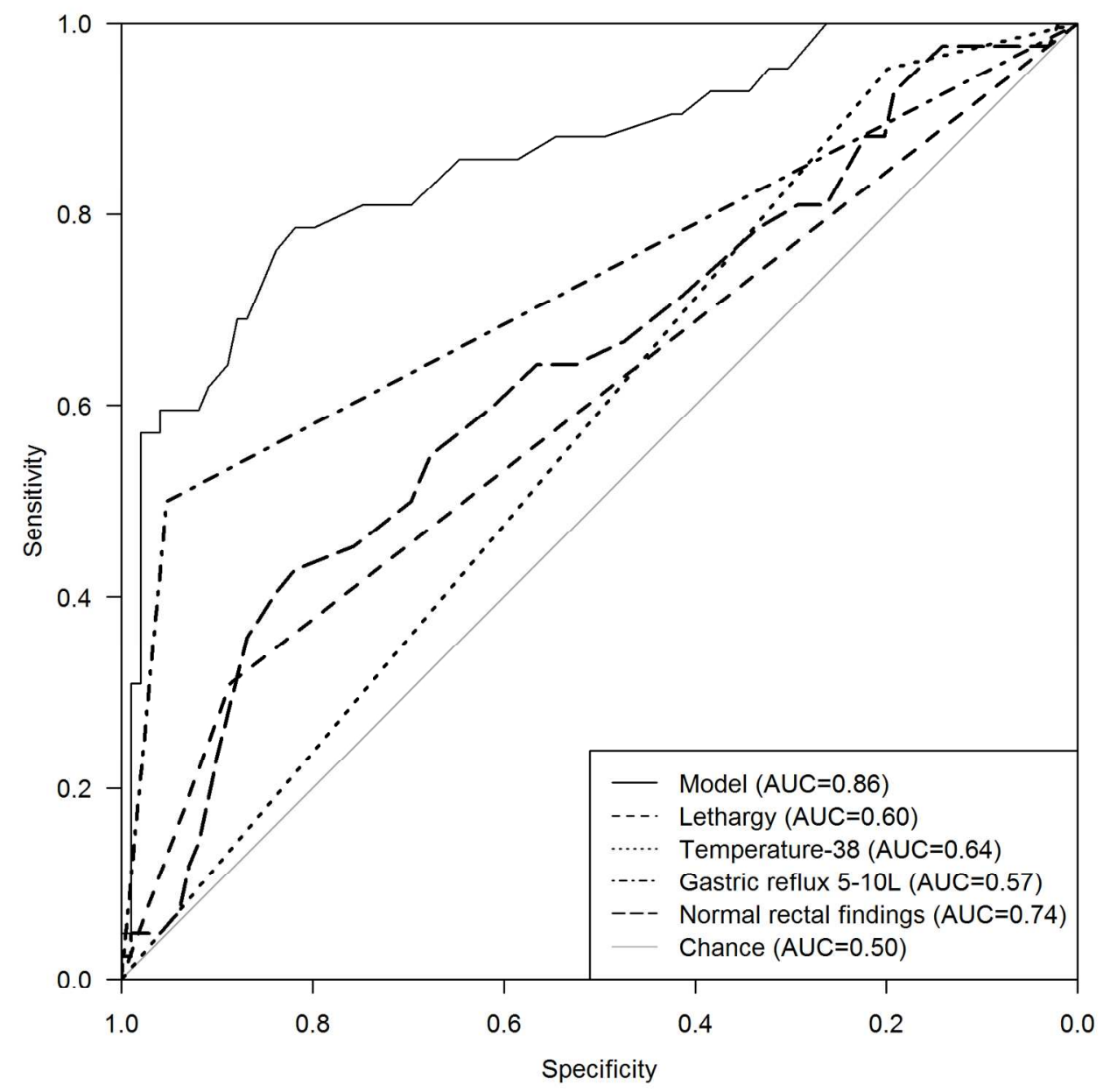

Figure $1 \mathrm{~A}$ ) The best model with clinical parameters predicting the probability of inflammatory colic in horses referred to a referral clinic (hospital 1 ) with acute, severe abdominal disease. The model included the clinical variables lethargy, rectal temperature above $38.0^{\circ} \mathrm{C}$, gastric reflux $5-10 \mathrm{~L}$, and normal rectal findings. With an area under the receiver operating characteristic (ROC) curve (AUC) of 0.86 the model correctly diagnosed inflammatory colic and surgical colic in $86 \%$ of the horses evaluated. $159 \times 159 \mathrm{~mm}(300 \times 300 \mathrm{DPI})$ 


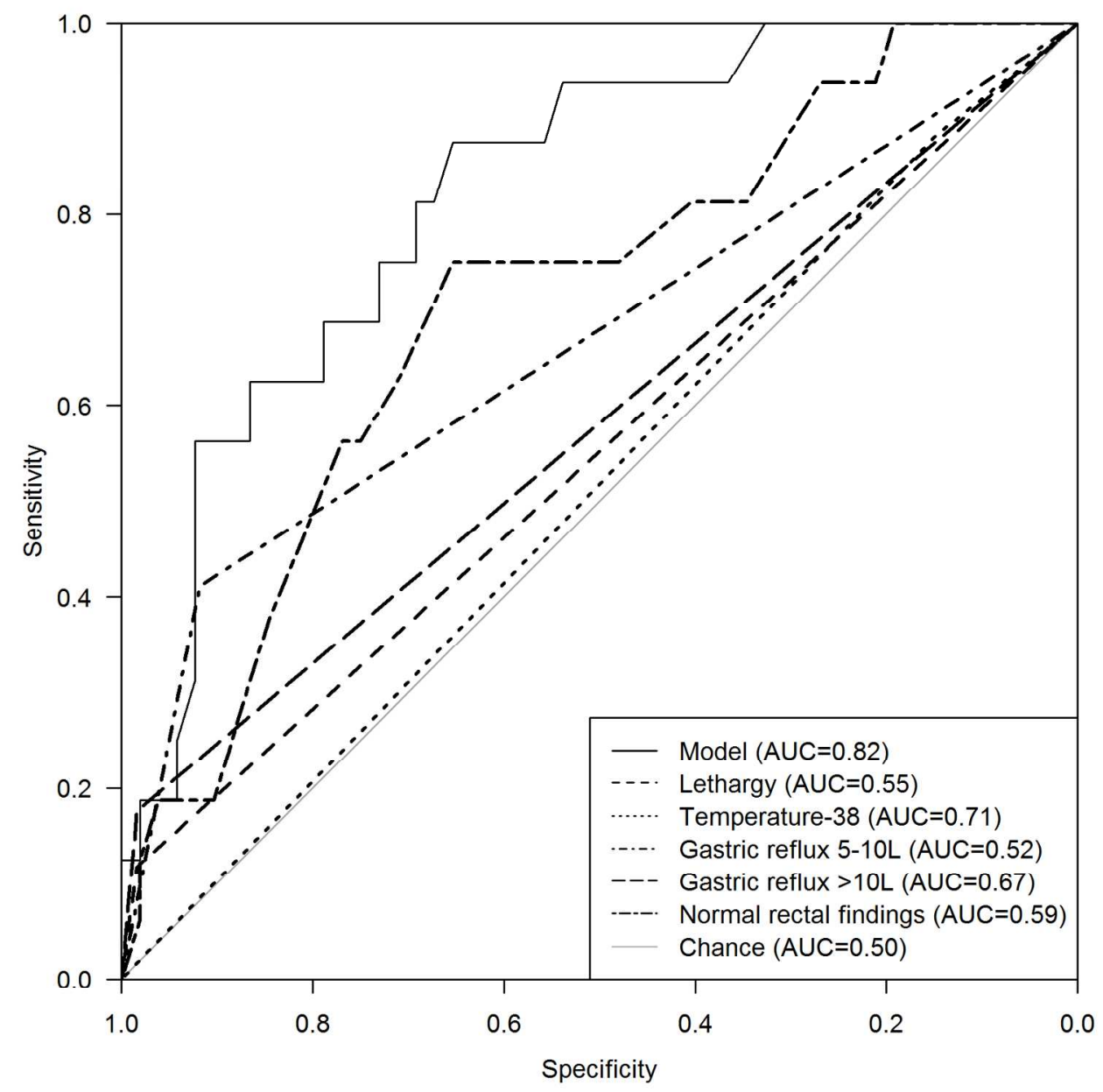

Figure 1B) Validation of the Clinical model in another referral clinic (hospital 2) identified the same variables as significant predictors (except gastric reflux, where large volume reflux ( $>10 \mathrm{~L}$ ) was superior to low volume reflux (5-10 L)). The AUC is 0.82 in hospital 2. $159 \times 159 \mathrm{~mm}(300 \times 300 \mathrm{DPI})$ 


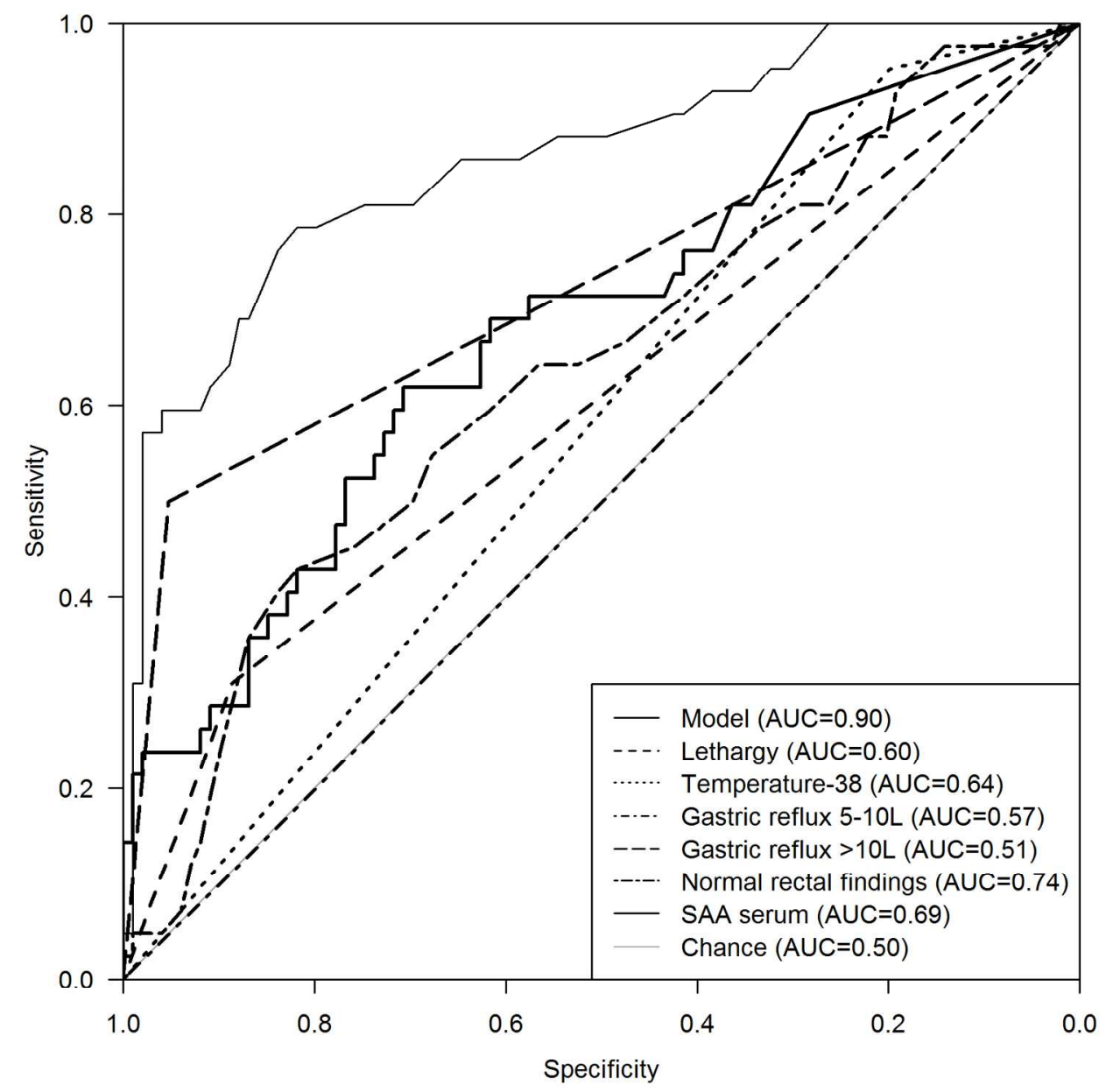

Figure 2A) The best model with clinical and blood parameters constructed in hospital 1 included only SAA in serum in addition to the clinical variables. SAA in serum improved the AUC to 0.90 from 0.86 . $159 \times 159 \mathrm{~mm}(300 \times 300 \mathrm{DPI})$ 


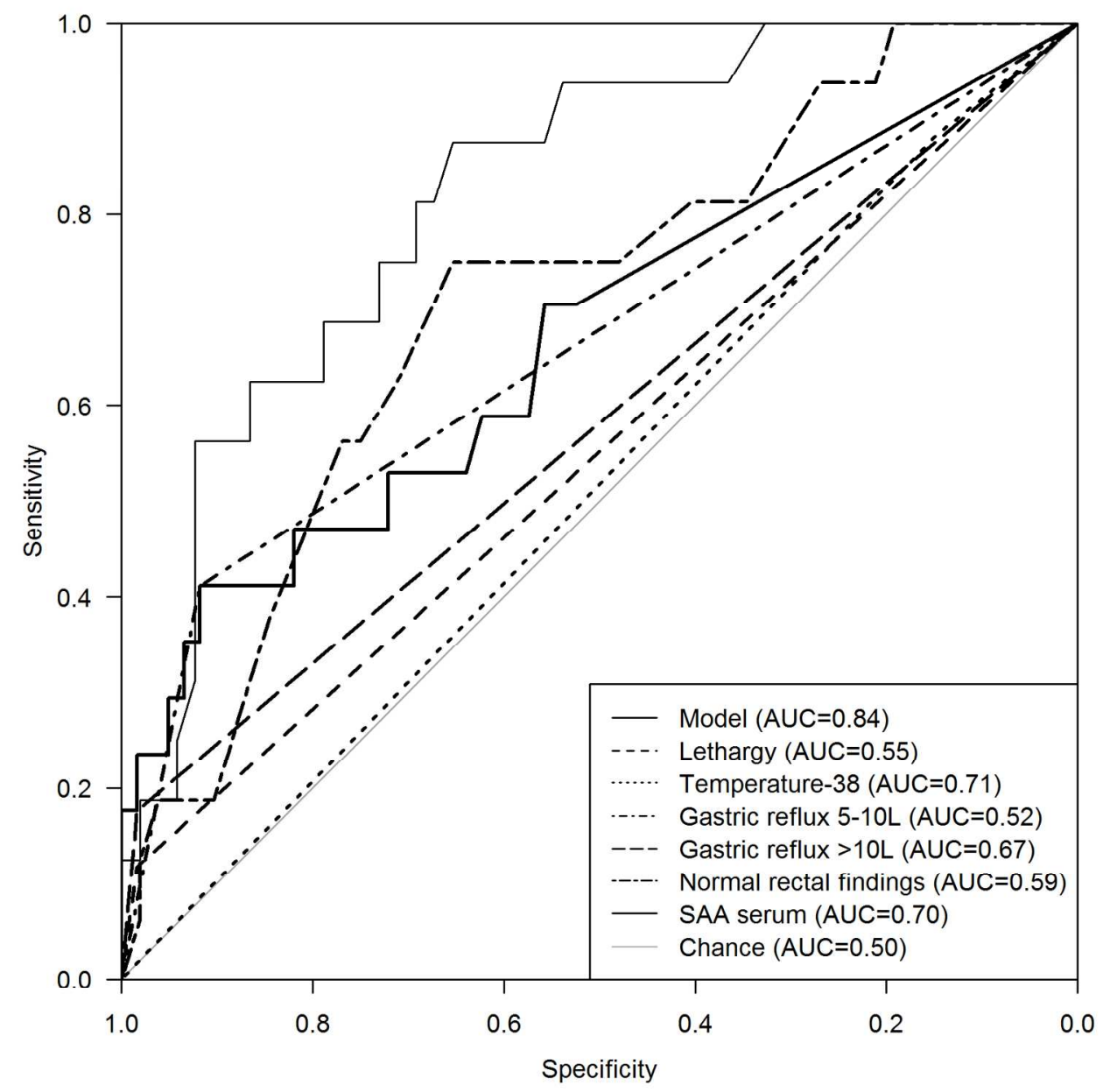

Figure 2B) SAA in serum likewise improved the clinical model in hospital 2 increasing the AUC to 0.84 from 0.82 . With SAA in serum in the model no further blood or PF variables significantly improved the model. $159 \times 159 \mathrm{~mm}(300 \times 300 \mathrm{DPI})$ 


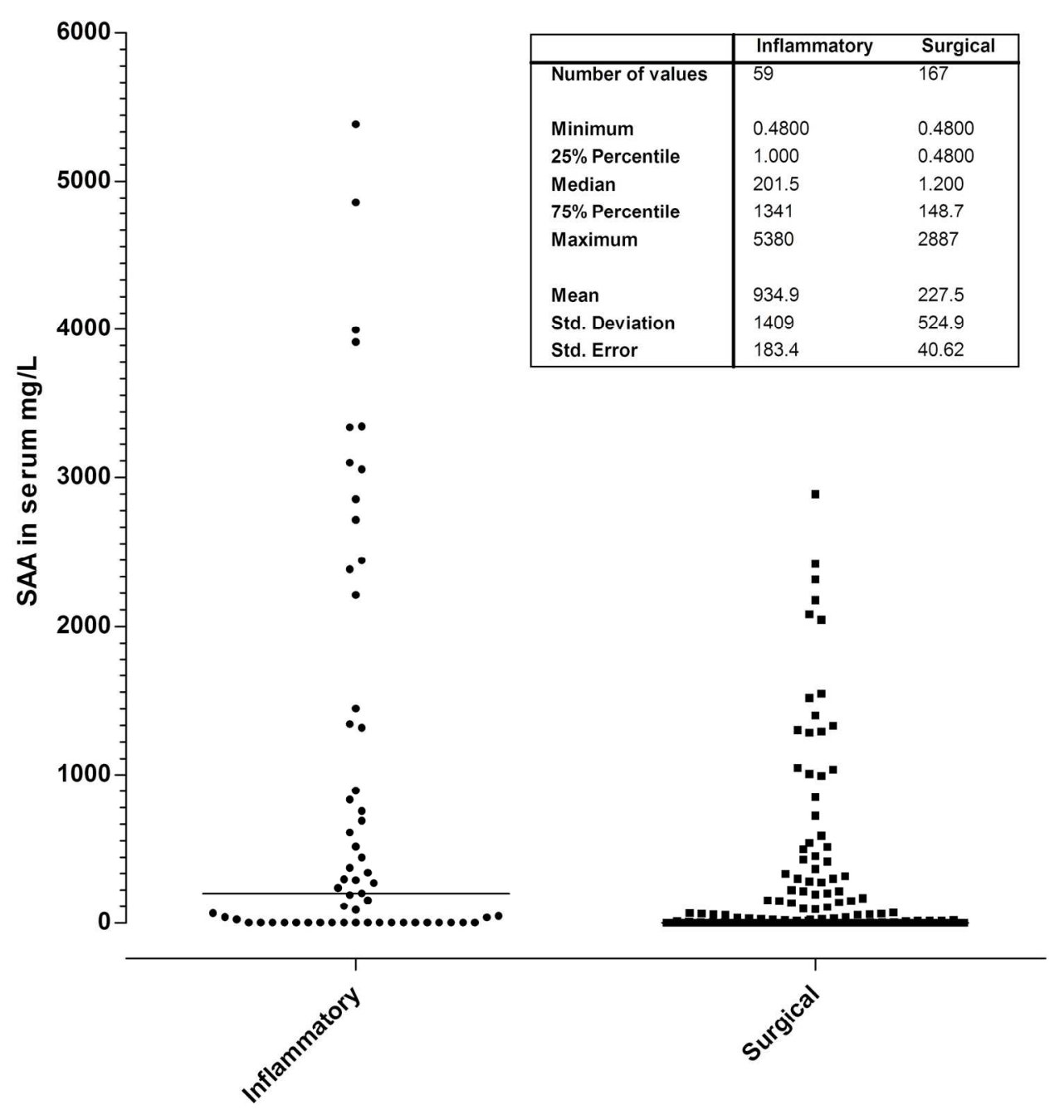

Serum amyloid A in serum from 226 horses with inflammatory and surgical colic. The horizontal line depicts the median.

$85 \times 91 \mathrm{~mm}(600 \times 600 \mathrm{DPI})$ 